\title{
Prognostic and therapeutic stratification through the PROFUND scale in patients with heart failure and comorbidities: PROFUND-IC registry
}

\author{
Beatriz Sánchez-Sauce ${ }^{*}$, Rosario Iguarán-Bermúdez², José L. García-Klepzig², Santiago Fernández², \\ Julia Barrado-Cuchillo², Miguel Villar-Martínez², Pablo Pérez-Mateo², Lidia López-Garcia3, \\ Francisco J. Martín-Sánchez ${ }^{4}$, Elpidio Calvo-Manuel2 ${ }^{2}$, Jesús Díez-Manglano ${ }^{5}$, Ignacio Vallejo-Maroto ${ }^{6}$, \\ Máximo Bernabeu-Wittel', Álvaro González-Franco ${ }^{8}$, Luis Matías Beltrán-Romero7, \\ José M. De-Miguel-Yanes ${ }^{9}$, Alejandra García-García ${ }^{9}$, Pau Llacer-Iborra ${ }^{10}$, Luis Manzano-Espinosa ${ }^{10}$, \\ José M. Casas-Rojo ${ }^{11}$, Emmanuel Andres ${ }^{12}$, Noel Lorenzo-Villalba ${ }^{12}$, and Manuel Méndez-Bailón ${ }^{2}$
}

${ }^{1}$ Department of Internal Medicine, Hospital Fundación de Alcorcón, Alcorcón; ${ }^{2}$ Department of Internal Medicine; ${ }^{3}$ Nursing School, and ${ }^{4}$ Department of Emergency, Hospital Clínico San Carlos, Universidad Complutense de Madrid, Madrid; ${ }^{5}$ Department of Internal Medicine, Hospital Royo Villanova, Zaragoza; ${ }^{6}$ Department of Internal Medicine, Hospital San Juan de Dios del Aljarafe, Sevilla; ${ }^{7}$ Department of Internal Medicine, Hospital Virgen del Rocío, Sevilla; ${ }^{8}$ Department of Internal Medicine, Hospital Universitario Central de Asturias, Asturias; ${ }^{9}$ Department of Internal Medicine, Hospital Gregorio Marañón, Madrid; ${ }^{10}$ Department of Internal Medicine, Hospital Ramón y Cajal, Madrid; ${ }^{11}$ Department of Internal Medicine, Hospital Infanta Cristina, Madrid. Spain; ${ }^{12}$ Internal Medicine Service, Diabète et Maladies Métaboliques, Hôpitaux Universitaires de Strasbourg, Strasbourg, France

\begin{abstract}
Introduction: The increase in life expectancy and the aging of the population are associated with an increase in the prevalence of chronic diseases. Comorbidities have an important impact on prognosis and functional capacity leading to a progressive deterioration of autonomy and quality of life and an increase in demand for medical care. Establishment of an accurate prognosis constitutes one of the primary objectives in healthcare. An accurate estimate of prognosis helps clinicians make diagnostic and therapeutic decisions, prevent iatrogenesis, and consider palliative care strategy as needed. It also allows the patient and family members to organize their preferences and priorities. Objective: To evaluate the PROFUND scale in patients with heart failure from a prognostic point of view. Methods: A multicenter cohort study including patients admitted for heart failure to internal medicine departments over a 6 -month period will be carried out. Inclusion criteria are patients with a diagnosis of heart failure and at least two criteria of multipathological patients and NT-proBNP $>1500 \mathrm{pg} / \mathrm{ml}$ upon admission. The PROFUND scale will be applied to all patients. Patients will be then stratified into four groups according to the PROFUND scale: low, moderate, moderate-high and high mortality risk. Conclusion: Our work is a prospective study that aims to apply the PROFUND scale to patients with heart failure in the hospital setting with the purpose of helping in decision-making with our patients, which could lead to improvements in the management of resources in our health system.
\end{abstract}

Key words: Heart failure. Comorbidity. Chronic disease.

Visual abstract available at https://spanishjmed.com/frame_esp.php?id=53

Correspondence:

*Beatriz Sánchez-Sauce

E-mail: beatrizsauce@gmail.com
Available online: 05-10-2021 Span J Med. 2021;1(3):136-141

www.spanishjmed.com 2696-5631 / @ 2021 Sociedad Española de Medicina Interna. Published by Permanyer. This is an open access article under the CC BY-NC-ND license (http://creativecommons.org/licenses/by-nc-nd/4.0/). 


\section{Introduction}

The prevalence and incidence of heart failure (HF) have been increasing in recent years as the population ages. The prevalence of HF is between 2 and $3 \%$, reaching $10-20 \%$ in patients between 70 and 80 years $^{1-3}$. This high prevalence creates an increase in the demand for health care, making HF the most important cause of admission to Spanish hospitals and representing $5 \%$ of all hospitalizations ${ }^{2,3}$. The frequency of hospital readmission for this disease ranges from $30 \%$ to $60 \%$ in the first 6 months following hospital discharge ${ }^{4}$. These patients show a different profile of comorbidity, which makes their care more complex. The comorbid conditions that are significantly increased include chronic renal failure, severe hematological disorders, malnutrition, psychiatric disorders, and pressure ulcers. These comorbidities could affect treatment and have an important prognostic impact leading to more hospital admissions, worsening quality of life, and increased mortality.

Most HF comorbidity registries tend to include conditions having a pathogenic relationship with HF and cardiovascular risk. Other conditions apparently unrelated to HF such as cognitive impairment, functional status, degenerative osteoarticular pathology, neoplastic processes, frailty, or socio-familial risk factors can directly influence the prognosis of these patients if they are not considered ${ }^{5}$. The RICA registry analyzed the prognostic value of baseline functional status, assessed by the Barthel index, showing a high prevalence of functional impairment in $55.9 \%$ of patients ${ }^{6}$. The pre-admission Barthel index was shown to be an independent predictor of short-term mortality.

It is in this context that the concept of pluripathology arises, identifying a population of patients with two or more chronic diseases with an equivalent degree of complexity. Pluripathology patients constitute a heterogeneous population with a series of easily identifiable common characteristics: greater complexity, clinical vulnerability, frailty, mortality, functional deterioration, polypharmacy, poorer health-related quality of life, and dependency ${ }^{7,8}$.

The prevalence of pluripathology patients in hospital setting varies between $25 \%$ and $50 \%$. HF is the most frequent defining category of pluripathology patients with a prevalence of $72-77 \%$. In addition, there is a high prevalence among pluripathology patients of other cardiovascular comorbidities related to HF (ranging from $30 \%$ to $70 \%)^{9}$ : arterial hypertension, diabetes, dyslipidemia, and atrial fibrillation.
In recent years, the PROFUND index, a specific tool for estimating the prognosis of mortality at 1 year in pluripathology patients, has been developed. This index was developed in a hospital-based multicenter cohort recruited in 36 Spanish hospitals. A total of 1632 patients were included and followed for 1 year and the index was subsequently derived and validated using standard methodology. This index stratifies 12-month mortality and is based on nine clinical, analytical, and socio-familial dimensions. The index stratifies pluripathology patients into four risk groups according to the score obtained, with mortality ranging from $12 \%$ to $14 \%$ in the lowest risk stratum to $61 \%$ to $68 \%$ in patients with 11 or more points ${ }^{8,9}$. However, this scale has not been evaluated in pluripathology patients with HF.

This study design aims to define the clinical characteristics of pluripathology patients with acute HF (AHF) and to prognostically stratify them using the PROFUND scale as well as to compare this stratification with other classic prognostic assessment scales in HF.

\section{Methodology}

\section{Design, study population, variables, data collection and analysis, ethical aspects, and limitations of the study}

\section{DESIGN AND STUDY POPULATION}

This is a nationwide prospective multicenter cohort study from the HF study group and the Pluripathologic Patients and Advanced Age group of the Spanish Society of Internal Medicine. All patients $>18$ years old admitted for HF as the main diagnosis in internal medicine services will be consecutively included over a period of 6 months-1 year. Inclusion and exclusion criteria are shown in table 1.

Table 1. Selection criteria for participating in the study

Inclusion criteria
- Patients admitted with a principal diagnosis of heart failure
- Patients with NT-pro-brain natriuretic peptide (NT-proBNP)
$\quad 300 \mathrm{pg} / \mathrm{mL}$ on admission and/or on arrival at the emergency
department
Exclusion criteria
- Signature of informed consent not obtained from either the
patient or legal representative


Table 2. PROFUND scale variables

\begin{tabular}{|l|l|}
\multicolumn{2}{|c|}{ PROFUND scale } \\
\hline Variable & Points \\
\hline
\end{tabular}

Age $>85$ years

Clinical features

- Active neoplasia

- Dementia

- NYHA functional class III-IV or mMRC 3-4

- Delirium during the last hospital admission

$\mathrm{Hb}<10 \mathrm{~g} / \mathrm{dL}$

Socio-familial features

- Barthel index $<60$

- Lack of caregiver or other than partner

$->4$ hospital admissions in the last 12 months

NYHA: New York Heart Failure Association Functional Classification. mMRC: modified Medical Research Council.

\section{Objectives}

The main objective of the study is to stratify pluripathology patients with over a 6-12 month period in hospitals of the Spanish National Health System using the PROFUND scale (Table 2). Secondary objectives include: to evaluate the epidemiological and clinical characteristics of pluripathology patients with HF as the primary diagnosis and 30-day mortality, early hospital readmission (< 30 days), and 1-year mortality; and to evaluate the destination of care of pluripathology patients with AHF after discharge.

\section{Data collection}

Data from patients admitted for AHF to the internal medical department will be prospectively collected by researchers (medical and nursing personnel). The following parameters will be collected: date of birth, sex, toxic habits, arterial hypertension, dyslipidemia, diabetes mellitus, degree of ventricular dysfunction (LVEF), NYHA dyspnea functional class at baseline (2 weeks before admission), presence of pluripathology (defined by the presence of two or more chronic diseases of the clinical categories listed in Table 3), NT-proBNP levels, Cancer Antigen 125 (CA-125), presence of $B$ lines on lung ultrasound, pleural effusion on chest $\mathrm{X}$-rays or
Table 3. Clinical categories for the identification of pluripathological patients

Clinical categories for the identification of pluripathological patients

\section{CATEGORY A}

A.1. Heart failure that in a clinically stable situation has been in NYHA II (symptoms with usual physical activity)

A.2. Ischemic cardiomyopathy

\section{CATEGORY B}

B.1. Vasculitis and systemic autoimmune diseases

B.2. Chronic kidney disease defined by glomerular filtration rate $<60 \mathrm{~mL} / \mathrm{m}$ or proteinuria persisting for 3 months

\section{CATEGORY C}

C.1. Chronic respiratory disease that in a situation of clinical stability presented with Grade II dyspnea mMRC3 (dyspnea at usual pace in flat), FEV1

\section{CATEGORY D}

D.1. Chronic inflammatory bowel disease

D.2. Chronic liver disease with signs of liver failure or portal hypertension

\section{CATEGORY E}

E.1. Stroke

E.2. Neurological disease with permanent motor deficit limiting basic daily living activities (Barthel index $<60$ )

E.3. Neurological disease with permanent cognitive impairment, at least moderate (Pfeiffer $\geq 5$ or more errors)

\section{CATEGORY F}

F.1. Symptomatic peripheral arterial disease

F.2. Diabetes mellitus with proliferative retinopathy or symptomatic neuropathy

\section{CATEGORY G}

G.1. Chronic anemia due to digestive losses or acquired hematologic disease

G.2. Active solid or hematologic neoplasm that does not require treatment with curative intent

\section{CATEGORY H}

H.1. Chronic osteoarticular disease that causes by itself a limitation for basic daily living activities (Barthel index $<60$ )

FEV1: force expiratory volume.

pulmonary echography, and inferior vena cava measurement by ultrasound. Data regarding the patient's HF history such as date of admission, date of readmission for HF, date of death, mortality due to cardiovascular and non-cardiovascular causes during follow-up, destination and treatment at discharge, will be collected.

Patients will be then stratified into four groups according to the PROFUND scale: 
- Patients at low mortality risk (0-2 points): those in whom the probability of mortality at 1 year is $<14 \%$

- Patients at moderate-low mortality risk (3-6 points): when the probability of mortality at 1 year is between 15 and $31 \%$

- Patients at moderate-high mortality risk (7-10 points): when the probability of mortality at 1 year is between 32 and $50 \%$

- Patients at high mortality risk ( $>11$ points): when the probability of mortality at 1 year is $>50 \%$.

In addition, data about all-cause mortality and readmissions at 30 days, 6 months, and 1 year will be collected. Participants will be assessed by the following scales: PROFUND scale, which stratifies multipathological patients into four mortality risk groups at admission; MEESSI scale to evaluate 30-day mortality in patients with the diagnosis of AHF; Meta-analysis Global Group in Chronic HF (MAGGIC) scale for the prediction of survival at 1 and 3 years; and Barthel index which evaluates functional capacity.

\section{Statistical analysis}

The sample size was estimated assuming a confidence level of $95 \%$, a precision of $5 \%$, a loss rate of $10 \%$, and a mortality rate at 12 months according to the data of the study on prognostic stratification and care approach for multipathologic patients of $13 \%$ for the low level, $26 \%$ for the low-intermediate level, $48 \%$ for the upper-intermediate level, and $64 \%$ for the high level. Based on these estimates, the expected number of patients would be 1,600 , about 400 patients per risk group.

A separate analysis of in-hospital and out-of-hospital mortality will be performed.

The analysis will be performed using the SPSS program.

\section{Ethical aspects}

The project will be submitted to the Clinical Research Ethics Committee of each hospital. All investigators and personnel involved in the project are aware of, and will respect, local, and international regulations in the field of ethical considerations for human experimentation including the Helsinki declaration with its revisions, the Belmont report, and other related documents.

Data confidentiality will be maintained in accordance with the Data Protection Law (Organic Law 5/92 of October 29 on the regulation of the automated processing of personal data, BOE October 30, 1992, modified by Organic Law 15/1999, of December 13, on the Protection of Personal Data and Law 41/2002, of November 14).

All patients, in addition to being informed verbally in detail about the project, will give their Informed Consent in writing before being included in the study.

\section{Discussion}

\section{Current status: rationale for a study of pluripathologic patients in AHF}

\section{IMPACT OF PLURIPATHOLOGY}

Pluripathology has an important impact on the prognosis of patients in primary and hospital care settings. It is estimated that the annual mortality of patients with multiple pathologies in primary care is around $6 \%$, while in hospitals it is around $20 \%$ during admission and $35 \%$ at 1 year. Mortality is significantly higher than in patients without multiple pathologies. Pluripathology also influences functional prognosis. These patients tend to deteriorate more during hospital admissions than non-pluripathological patients and most of them do not recover to their baseline functional state by the time of discharge. In addition, the group of patients with pluripathology presents a particular susceptibility and clinical fragility that leads to frequent demand for care due to exacerbations, worsening the patient's condition with a progressive deterioration of their autonomy and quality of life ${ }^{9}$.

\section{NeEd fOR PROGNOSTIC STRATIFICATION}

$\mathrm{HF}$ is one of the main causes of death in the general population, but the prognosis varies widely depending on the patient. Quantification of the risk of these patients could improve and individualize the treatment, as well as help establish a therapeutic care plan. It is important for the patient and the family to understand the most accurate estimate of prognosis possible, leading to an appropriate choice of diagnostic, and therapeutic approaches and optimized planning of health care among HF patients ${ }^{5,6}$.

For example, scales such as the MEESSI-AHF can be used to predict 30-day mortality in patients presenting to emergency departments with a diagnosis of $\mathrm{AHF}^{10}$. It can be very useful in estimating the prognosis of these patients and can help making clinical decisions such as whether to admit or discharge a patient based on the risk of dying in the next 30 days. The MEESSI-AHF score was obtained in 4,867 consecutive patients with AHF admitted 
to Spanish emergency departments during 2009 and 2011 and was subsequently validated in 3,229 patients with AHF collected in 2014 (ST-elevation acute coronary syndrome patients were excluded from the study).

The MEESSI-AHF risk model includes 13 variables readily available in emergency departments. In some patients for whom troponin, NT-pro-brain natriuretic peptide, and/or baseline Barthel are unknown, the risk model can also be applied. Forty percent of patients classified as low risk (30-day mortality $<2 \%$ ) should be considered potential candidates for discharge from the emergency department, without requiring admission, after an adequate response to initial treatment. The $10 \%$ of patients classified as very high risk (30-day mortality $>2 \%$ ) may clearly benefit from hospital admission. The MEESSI-AHF risk scale may be found at the following link: http://meessi-ahf.risk.score-calculator-ica-semes.portalsemes.org.

However, the use of prognostic tools in this population is scarcely used. Among the scales related to cardiac dysfunction, the MAGGIC scale has shown the best accuracy. The MAGGIC scale is the result of the trial MetaAnalysis Global Group in Chronic HF (MAGGIC) ${ }^{11}$. The meta-analysis included 39,372 patients extracted from 30 studies (six randomized clinical trials and 24 observational registries), with a mean follow-up of 2.5 years (1.0-3.9) during which $40.2 \%$ of deaths occurred.

The risk predictor variables are age (for each 10-year increase), male sex, body mass index (for each $1 \mathrm{~kg} / \mathrm{m}^{2}$ increase above $30 \mathrm{~kg} / \mathrm{m}^{2}$ ), smoking, diabetes mellitus, NYHA functional Classes III and IV, left ventricular ejection fraction $\leq 40 \%$, chronic obstructive pulmonary disease, HF diagnosed more than 18 months ago, and renal failure (for each $0.11 \mathrm{mg} / \mathrm{dL}$ increase above $3.97 \mathrm{mg} / \mathrm{dL}$ creatinine). The protective factors are systolic blood pressure (for each increase of $10 \mathrm{mmHg}$ ), NYHA I HF, and treatment with beta-blockers, angiotensin converting enzyme inhibitors, or angiotensin II receptor antagonist. When entering these values to establish the risk score, not all factors add 1 point of risk, but most add between 2 and 3 points, with a maximum of 15 points (those patients over 80 years of age and LVEF $\leq 40 \%$ ). It is important to note that when several of the factors are individually combined, the risk is not simply added, but multiplied. Based on this risk scale, a probability of death at 1 year and at 3 years is generated, depending on the patient's characteristics. The MAGGIC scale is available on the internet (www.heartfailurerisk.org).

However, the reality is that most physicians do not use these scales in routine clinical practice. This is due to several reasons. First, we know that their accuracy at the individual level and in the short term is low; second, specific medical management according to patient risk has not been defined (with the exception of cardiac transplantation/ventricular assist devices); and finally, they do not usually include variables of significant importance in clinical practice such as comorbidity, frailty, functionality, or socio-affective type.

In patients with HF, comorbidities are often the cause of hospital readmission. In fact, in some series it has been observed that only one third or less of readmissions are due to HF per se ${ }^{12}$. This occurs more frequently in patients with HF with preserved ejection fraction, among whom mortality is often linked to non-cardiovascular diseases such as neoplasms, renal disease, or infections ${ }^{13}$. Taking into consideration the importance of comorbidities in morbidity and mortality of patients with $\mathrm{HF}$, special attention has recently been drawn to the importance of a more global approach to HF patients rather than a restrictive assessment (i.e., by focusing exclusively on cardiac disease). This has led to the assessment of comorbidity acquiring greater prognostic importance in patients with HF.

The Charlson score is currently the most widely used instrument for prognostic assessment in patients with comorbidity ${ }^{14}$. It is a numerical summative scale, so that patients with higher scores are more likely to die at 12 months. Mortality by quartiles of the patients studied was as follows: score $0,12 \%$; score $1-2,26 \%$; score $3-4,52 \%$; and score $>5,85 \%$. The prognostic value of the Charlson score as an independent predictor of mortality or readmission at 1 year has also been confirmed in the RICA registry ${ }^{15}$.

The PROFUND index 8,9 is a specific tool for estimating the 1-year mortality in multipathological patients. This index was developed in a multicenter hospital-based cohort recruited in 36 Spanish hospitals. A total of 1,632 patients were included and followed for 1 year and the index was subsequently derived and validated using standard methodology. This index stratifies mortality at 12 months and is based on nine clinical, analytical, and socio-familial dimensions. The index stratifies patients with multiple pathologies into four risk groups according to the score ranges obtained, with mortality ranging from $12 \%$ to $14 \%$ in the lowest risk stratum to $61 \%$ to $68 \%$ in those with 11 or more points. It has also shown to be useful in hospitalization areas outside internal medicine ${ }^{16}$ (Cardiology Hospitalization Units) and in primary care ${ }^{17}$ where the scoring ranges were recalibrated.

The use of prognostic indices will allow us to classify patients with HF into groups according to the estimated 
risk of mortality, which is an important aid for improving health planning and decision making. Thus, we present this study with the aim of providing a tool, through the PROFUND scale, that stratifies patients with HF according to their prognosis, and thus guides decision-making and health planning for these patients.

\section{Conclusion}

Our work is a prospective study that aims to apply the PROFUND scale to patients with HF in the hospital setting with the purpose of helping in decision-making with our patients, which could lead to improvements in the management of resources in our health system.

\section{Funding}

This research was funded by Sociedad Española de Medicina Interna.

\section{Conflicts of interest}

The authors declare that they have no conflicts of interest.

\section{Ethical disclosures}

Protection of human and animal subjects. The authors declare that no experiments were performed on humans or animals for this study.

Confidentiality of data. The authors declare that they have followed the protocols of their work center on the publication of patient data.

Right to privacy and informed consent. The authors have obtained the written informed consent of the patients or subjects mentioned in the article. The corresponding author is in possession of this document.

\section{References}

1. Cortina A, Reguero J, Segovia E, Lambert JL, Cortina R, Arias JC, et al. Prevalence of heart failure in Asturias (a region in the North of Spain). Am J Cardiol. 2001;87:1417-9.

2. Banegas JR, Rodríguez-Artalejo F, Guallar-Castillón P. Situación epidemiológica de la insuficiencia cardiaca en España. Rev Esp Cardiol Supl. 2006;6:4C-9C

3. Fernández Gassó ML, Hernando-Arizaleta L, Palomar-Rodríguez JA Soria-Arcos F, Pascual-Figal DA. Trends and characteristics of hospitalization for heart failure in a population setting from 2003 to 2013. Rev Esp Cardiol (Engl Ed). 2017;70:720-6.

4. Dharmarajan K. Comprehensive strategies to reduce readmissions in older patients with cardiovascular disease. Can J Cardiol. 2016; 32:1306-14.

5. Conde-Martel A, Hernández-Meneses M. Prevalencia y significado pronóstico de la comorbilidad en la insuficiencia cardiaca. Rev Clin Esp (Barc). 2016;216:222-8.

6. Formiga F, Chivite D, Conde A, Ruiz-Laiglesia F, Franco ÁG, Bocanegra $\mathrm{CP}$, et al. Basal functional status predicts three-month mortality after a heart failure hospitalization in elderly patients-the prospective RICA study. Int J Cardiol. 2014;172:127-31.

7. García-Morillo JS, Bernabeu-Wittel M, Ollero-Baturone $M$ Aguilar-Guisad M, Ramírez-Duque N, de la Puente MA, et al. Incidencia y características clínicas de los pacientes con pluripatología ingresados en una unidad de medicina interna. Med Clin (Barc). 2005;125:5-9.

8. Bernabeu-Wittel M, Ollero-Baturone M, Moreno-Gaviño L, Barón-Franco B, Fuertes A, Murcia-Zaragoza J, et al. Development of a new predictive model for polypathological patients. The PROFUND index. Eur J Intern Med. 2011:22:311-7.

9. Bernabeu-Wittel M, Barón-Franco B, Nieto-Martín D, Moreno-Gaviño L, Ramírez-Duque N, Ollero-Baturone M. Estratificación pronóstica y abordaje asistencial de los pacientes pluripatológicos. Rev Clin Esp (Barc). 2017:217:410-9.

10. Miró Ò, Rossello X, Gil V, Martín-Sánchez FJ, Llorens P, Herrero-Puente P et al. Predicting 30-day mortality for patients with acute heart failure in the emergency department: a cohort study. Ann Intern Med. 2017;167:698.

11. Pocock SJ, Ariti CA, McMurray JJ, Maggioni A, Køber L, Squire IB, et al. Predicting survival in heart failure: a risk score based on 39372 patients from 30 studies. Eur Heart J. 2013;34:1404-13.

12. Dunlay SM, Redfield MM, Weston SA, Therneau TM, Hall Long K, Shah ND, et al. Hospitalizations after heart failure diagnosis a community perspective. J Am Coll Cardiol. 2009;54:1695-702.

13. Ather S, Chan W, Bozkurt B, Aguilar D, Ramasubbu K, Zachariah AA, et al. Impact of noncardiac comorbidities on morbidity and mortality in a predominantly male population with heart failure and preserved versus reduced ejection fraction. J Am Coll Cardiol. 2012;59:998-1005.

14. Charlson ME, Pompei P, Ales KL, MacKenzie CR. A new method of classifying prognostic comorbidity in longitudinal studies: development and validation. J Chronic Dis. 1987;40:373-83.

15. Ruiz-Laiglesia FJ, Sanchez-Marteles M, Perez-Calvo Jl, Formiga F, Bartolome-Satue JA, Armengou-Arxe A, et al. Comorbidity in heart failure. Results of the Spanish RICA registry. QJM. 2014;107:989-94.

16. López-Garrido MA, Martín-Portugués IA, Becerra-Muñoz VM, Orellana-Figueroa HN, Sánchez-Lora FJ, Morcillo-Hidalgo L, et al. Prevalencia de pluripatología y valor pronóstico del índice PROFUND en una unidad de hospitalización de Cardiología. Rev Clin Esp (Barc). 2017;217:87-94.

17. Colombo PB, Martín MD, de la Pisa BP, Lozano MJ, Camúñez MA, Wittel MB. Validación de un modelo pronóstico para pacientes pluripatológicos en atención primaria: estudio PROFUND en atención primaria. Aten Primaria. 2014;46 Suppl 3:41-8. 